\title{
Las ciudades rurales hoy y cómo hacer frente a los megaproyectos que vendrán en el futuro
}

Miguel Pickard

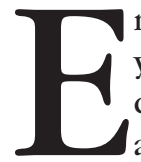

n primer lugar quisiera agradecer al doctor Raymundo Sánchez Barraza y al Cideci por brindarnos la posibilidad de hacer esta presentación del libro Depredación en el horario del seminario de los jueves. Me atrevo a decir que todas las personas que fuimos convocadas a presentar el libro estamos de acuerdo en que este hermoso recinto es el mejor lugar para la presentación de esta obra por la diversidad del público que aquí suele reunirse los jueves.

A título personal, quisiera agradecer en especial a la profesora de la Universidad Nacional Autónoma de México (UNAM) Mariflor Aguilar, quien me ha ofrecido muchas oportunidades para compartir con el seminario "Democracia y territorio" de la Facultad de Filosofía y Letras de la UNAM algunas ideas construidas en colectivo, y otras personales, sobre las ciudades rurales.

En los próximos minutos quisiera compartir con ustedes algunas reflexiones sobre las ciudades rurales construidas en el sexenio del gobernador Juan Sabines Guerrero. En varios momentos de este año 2013, varias amistades, tanto de México como del extranjero, se han acercado para preguntar, ¿qué hay de las ciudades rurales? y ¿qué noticias hay de ellas en este nuevo sexenio de Manuel Velazco Coello? Mi respuesta siempre ha sido: las ciudades rurales están muertas. Como idea, como concepto, ya murieron. El gobierno del Güero no las va a asumir, no las va a promover, no las va a financiar. Es más, ni siquiera se ha pronunciado sobre las ciudades rurales, no ha mencionado qué destino tendrán. Su silencio al respecto habla enormidades sobre un proyecto que heredó del gobierno anterior y que fue el proyecto estrella del gobierno de Sabines.

Si bien es cierto que el gobierno del Güero no se ha posicionado con claridad respecto a las ciudades rurales, hemos descubierto en días recientes que las ciudades rurales no están muertas. Al contrario, gracias a la visita que pudimos realizar varias personas aquí presentes a las dos ciudades rurales más 
nuevas, Ixhuatán y Jaltenango, inauguradas días antes de que terminara el mandato de Juan Sabines, tenemos que decir que las ciudades rurales no están muertas, tienen vida, tienen movimiento. Así como las ciudades rurales han integrado y transformado a cientos de familias de la zona norte, en el caso de Ixhuatán, y cientos de familias de La Frailesca en el caso de Jaltenango, también es justo decir que estas mismas familias campesinas han incorporado y transformado a las ciudades rurales. Ha sido un proceso dinámico en al menos esos dos sentidos: las ciudades rurales han transformado a los campesinos y los campesinos han transformado a las ciudades.

De nuestra reciente visita a las dos ciudades rurales mencionadas, creo que el consenso de los que fuimos es que hay algunos aspectos que se salvan. No voy a profundizar al respecto aquí, porque creo que, en los minutos siguientes, las compañeras y los compañeros que fuimos a las dos ciudades podrán aportar más, en términos positivos o negativos, de lo que vimos.

¿Quiere decir esto que terminamos concluyendo que las ciudades rurales son buenas? y ¿que han sido un aporte positivo para las familias campesinas de Chiapas y que se deberían construir más ciudades rurales en el resto del estado y tal vez extender esta experiencia a otras fronteras?

En absoluto. La respuesta es un rotundo no. El hecho de que algunos aspectos de las ciudades rurales estén en marcha y que incluso hayan resultado funcionales para las familias, no implica un respaldo al concepto de ciudades rurales. Creo que ése es el consenso de quienes visitamos Ixhuatán y Jaltenango ayer y antier. Sí, algunas cosas, como la cercanía de las escuelas para los y las jóvenes, se salvan, pero las ciudades rurales siguen siendo una aberración, una pesadilla, que de ninguna forma debería repetirse, al menos así como se han realizado hasta el momento.

Una de las razones, entre las muchas que podríamos dar, de nuestro rechazo, o al menos de mi rechazo, al concepto de ciudades rurales es que es una idea, una construcción impuesta a las familias campesinas. Las ciudades rurales de Chiapas son el resultado de ideas pensadas en otros lugares, en el Banco Mundial, en el Banco Interamericano de Desarrollo, en el gobierno de México, e impuestas y hechas realidad por el gobierno de Chiapas.

Pero de ninguna manera representan la voz de las y los campesinos e indígenas de Chiapas. Las ciudades rurales de ninguna manera son el resultado de consultas realizadas a nivel de base con la gente, y por ello, porque no incorporan el sentir de los pueblos rurales, no son la expresión de cómo la gente del campo quisiera mejorar su vida. Desgraciadamente, los pueblos rurales de Chiapas siguen siendo objetos, objetos de los planes de organismos y de gobiernos extranjeros y nacionales, objetos de manipulación, objetos de experimentación social, y en este marco de los megaproyectos nunca son sujetos, nunca sujetos de su propio destino. 
Hasta que los pueblos de Chiapas, todos los pueblos de Chiapas, sean sujetos de su destino, como lo han hecho dignamente los zapatistas, los proyectos como las ciudades rurales seguirán siendo proyectos fallidos que benefician a unas pocas personas en el gobierno y a unas pocas empresas.

Entonces, ¿cuál es la situación actual de las ciudades rurales? Revisemos las cuatro ciudades rurales existentes. En la primera, Nuevo Juan de Grijalva, podemos decir que las casas ahí, mal construidas, con defectos de diseño y de materiales, y con despojo de tierras de por medio, hicieron frente a una emergencia provocada por las lluvias torrenciales y el desgajamiento de cerros en 2007. En Santiago El Pinar, encontramos el fracaso absoluto de este proyecto. La única ciudad rural construida en una zona indígena, yo la llamo la ciudad del racismo caxlán, porque ahí se construyeron las casas más pequeñas, con los peores materiales de construcción, en el peor lugar, en condiciones precarias y con un descuido total y absoluto de la cultura indígena. Prueba de su fracaso es su actual estado de abandono casi total.

En Ixhuatán y en Jaltenango encontramos otra historia: casas ligeramente más grandes y más funcionales que en las otras dos ciudades, construidas con materiales de más alta calidad, estéticamente menos chocantes, y, lo más importante, en estas dos ciudades rurales de más reciente inauguración -en ambos casos menos de un año de existencia- no hubo presión para que los campesinos dejaran sus tierras tradicionales a cambio de ir a vivir en las ciudades. Las campesinas y los campesinos siguen teniendo sus tierras, las siguen trabajando y, de hecho, uno de los campesinos en Jaltenango confirmó una de nuestras ideas: son las familias campesinas las que están subsidiando, con su trabajo en el campo, con sus cosechas, con su maíz, su frijol, su café y otros productos, la vida en esas ciudades rurales.

En Ixhuatán y en Jaltenango, el gobierno de Chiapas ya no está montando proyectos productivos, ya no pone ensambladoras de "economía ficción" como la que existió en Santiago El Pinar y que hoy se encuentra cerrada. En estas ciudades rurales de Ixhuatán y Jaltenango, ahora y como siempre, es la tierra de las familias campesinas, la que les está dando sus ingresos, la que les está dando de comer, la que les está permitiendo comprar otros productos necesarios que las familias campesinas no producen.

Esto no quiere decir que haya suficiente trabajo. No hay el trabajo suficiente para emplear a todas las personas que quisieran trabajar. Todavía hay necesidad de que al menos unos miembros de la familia migren a otras ciudades, a otros estados, y seguramente a Estado Unidos, en busca de empleo. Pero al menos en Ixhuatán y en Jaltenango, la gente sigue teniendo su tierra y el gobierno ya no está obligando a la gente a salir de ella, sino que, al contrario, para que esas dos ciudades rurales tengan un poco de vida, un poco de sostenibilidad económica, las familias campesinas están trabajando en sus tierras y ganando 
un poco de ingresos. Por eso decimos que las familias están subsidiando su vida en esas ciudades rurales.

Entonces, ¿en qué quedamos con las ciudades rurales? En el mejor de los casos, las familias campesinas, por ejemplo en Ixhuatán y Jaltenango, han refuncionalizado a las ciudades rurales, las han adaptado a sus vidas, y han aceptado algunas de sus modalidades, como la cercanía de escuelas para los hijos e hijas y, en algunos casos, de clínicas para la población entera.

Pero, nuevamente, ¿son las ciudades rurales la solución de los muchos problemas, principalmente económicos, que padecen las familias campesinas? Yo, y creo que casi todos y todas, si no todos y todas, los que estamos en esta presentación, decimos que no. Las ciudades rurales fueron implementadas para hacer fluir las millonadas de dinero federal hacia Chiapas y hacia los bolsillos de los malos gobernantes, de los cuates de éstos, de sus empresas y de las empresas extranjeras, con muy pocos, o nulos, beneficios para las familias campesinas e indígenas de Chiapas. Este proyecto de ciudades rurales, como muchos otros megaproyectos, no están pensados para el desarrollo de los pueblos. Si la idea fuera aportar para el desarrollo de los pueblos, lo principal, lo básico, lo democrático sería tomar en cuenta la palabra de los directamente afectados. Y, obviamente, eso no pasó.

Entonces ¿están muertas las ciudades rurales de Chiapas? Pues sí y no: una, Santiago El Pinar, sí está muerta, sin vida alguna; otra, Nuevo Juan de Grijalva, medio muerta porque no tiene vida económica autónoma; y dos más, Ixhuatán y Jaltenango, que ahí van, lejos de las intenciones originales de los funcionarios de desplazar a las familias campesinas de sus tierras y que ahora funcionan más como unidades habitacionales de las cabeceras del municipio de Ixhuatán y del municipio de Ángel Albino Corzo, respectivamente. Pero en ningún caso representan lo que los pueblos mismos hubieran deseado como alternativas a su situación histórica de pobreza y marginación.

Creo que lo importante de esta revisión rápida a la situación de este megaproyecto de las ciudades rurales supuestamente sustentables es preguntarnos ¿y ahora qué viene? El actual gobernador, el Güero Velasco, no ha dicho nada, o casi nada, respecto a las ciudades rurales. Pero lo que no podemos dudar es que, de tener los fondos para lanzar otro megaproyecto, este gobierno, o el siguiente, lo hará. Sí, ¿ahora qué? ¿Ahora qué proyecto millonario se impulsará? ¿Otra vez se tratará de construir la autopista de San Cristóbal a Palenque, desplazando a los pueblos indígenas que habitan en la ruta y que tienen otros planes para sus tierras?, ¿tal vez la autopista de San Cristóbal a la frontera con Guatemala?, ¿quizá a hacer de Chiapas un nuevo Cancún turístico, mediante la construcción de aeropuertos, hoteles, canchas de golf, que también intentará desplazar a los campesinos y a los pueblos indígenas de sus tierras?, ¿vendrán las empresas extranjeras contaminantes a pintarse el rostro de verde 
mediante la compra y venta de bonos de carbono basados en los bosques de Chiapas? o ėvendrá algo que todavía no nos podemos imaginar, pero que se está craneando en alguna oficina gubernamental o en las oficinas de alguna empresa transnacional?

De que vendrán más planes, y más obras y más megaproyectos, no cabe duda. Lo importante es que los pueblos se preparen y se defiendan. Revisando los diferentes megaproyectos que se han realizado desde 2001, cuando Vicente Fox echó a andar el Plan Puebla Panamá, podemos decir que los pueblos de Mesoamérica hicieron con los megaproyectos del Plan Puebla Panamá lo que los campesinos y las campesinas de Chiapas han hecho con los megaproyectos que aquí se han impulsado: a lo largo de la geografía de Mesoamérica, las grandes obras han sido detenidas, postergadas, desviadas, alteradas y canceladas, gracias a las protestas y a las movilizaciones populares.

Mirando hacia el futuro, y hacia los megaproyectos que los poderosos tratarán de impulsar, creo que podemos aplicar algunas lecciones que nos han dado los compañeros y compañeras de Mesoamérica en su defensa de sus tierras y de sus territorios. Quisiera rápidamente recuperar la experiencia del pueblo de Atenco, que en 2001 fue amenazado por el megaproyecto más importante del sexenio de Fox, la construcción de un nuevo aeropuerto para la ciudad de México.

Quiero dejar, a manera de conclusión, unas ideas de por qué triunfó el movimiento de Atenco contra el aeropuerto de México. La resistencia levantada en Atenco, al ocurrir en el primer año del gobierno de Fox, fue emblemática para las organizaciones sociales en otros lugares, que vieron en ella una fuente de inspiración para sus propias luchas. Además, la resistencia de Atenco dio pautas de lo que puede ser una defensa territorial con posibilidades de triunfo. Esas condiciones en Atenco consistieron en lo siguiente:

1. Una base social informada y consciente de las implicaciones de los planes hechos por los gobiernos y las empresas transnacionales.

2. La rápida y eficaz organización y movilización de la base (el mismo día de la expropiación se tomaron carreteras en protesta).

3. La determinación de no ceder, no transigir; no es no.

4. La voluntad de persistir en la lucha "hasta las últimas consecuencias" (simbolizada por los machetes desenfundados);

5. La implementación de medidas de autodefensa (los comuneros cavaron zanjas para impedir el paso de trascabos y realizaron rondines continuos por el perímetro de las terrenos amenazados).

6. El uso efectivo de los medios de comunicación, en especial de los alternativos.

7. La preparación consensuada de propuestas y alternativas. 
Si recordamos el ejemplo de Atenco, y los ejemplos que los y las zapatistas han dado en tantas ocasiones, los pueblos podrán no sólo defenderse de los planes de los malos gobiernos, sino que, frente a los megaproyectos que los tratan como meros objetos, podrán ser los sujetos de su futuro y de su desarrollo -un desarrollo incluyente y popular. 